\title{
Mechanical Bowel Preparation: Keep It or Abandon It?
}

\author{
Hungdai Kim \\ Department of Surgery, Gastrointestinal Cancer Center, Kangbuk Samsung Hospital, Seoul, Korea
}

\section{See Article on Page 161-167}

Until recently, vigorous preoperative mechanical cleansing of the bowel, together with or without the use of oral antibiotics, was thought to reduce the risk of septic complications after colorectal surgery. However, well-designed clinical trials regarding mechanical bowel preparation (MBP) started to be published from 1972, and their results caused some colorectal surgeons to doubt this long-standing custom. From 2003, Guenaga et al. [1], using the Cochrane database, reported four times on the use of MBP for elective colorectal surgery. They concluded that there was no statistically significant evidence that patients benefited from MBP or from the use of rectal enemas.

However in a 2003 survey, the American Society of Colon and Rectal Surgeons estimated that $99 \%$ of its members still prescribed some type of MBP and that $75 \%$ used oral antibiotic prophylaxis as part of their standard preoperative protocol for elective colorectal surgery [2]. Also, the authors revealed that most of the respondents (90\%) from a Korean national survey were in favor of a preoperative MBP procedure and that $49 \%$ of the respondents agreed with the use of oral antibiotics [3]. Despite of the many studies insisting that MBP has no usefulness, current clinical practice does not follow any guideline. This may be explained as the 'no more MBP' concept being beyond surgeons' common sense feeling that the surgical field should be protected from contamination sources to prevent infection-related complications.

There are several practical issues concerning the MBP procedure. Firstly, MBP procedures vary among clinicians. Thus, standardization for the MBP procedure is very much needed. Secondly, novel bowel preparation agents, Coolprep (polyethylene glycol solution + ascorbic acid), Picolyte (sodium picosulphate +

Correspondence to: Hungdai Kim, M.D.

Department of Surgery, Kangbuk Samsung Hospital, 29 Saemunan-ro, Jongno-gu, Seoul 110-746, Korea

Tel: +82-2-2001-8335, Fax: +82-2-2001-2131

E-mail: hungdai.kim@samsung.com

(C) 2013 The Korean Society of Coloproctology

This is an open-access article distributed under the terms of the Creative Commons Attribution NonCommercial License (http://creativecommons.org/licenses/by-nc/3.0) which permits unrestricted noncommercial use, distribution, and reproduction in any medium, provided the original work is properly cited. magnesium citrate), have been introduced into clinical practice from 2012. These agents reduced the inconvenience to the patients. However, the effectiveness and safety of MBP using these agents for colon surgery are not well known. Despite the availability of several different products for colon cleansing, a need for a better regimen that provides excellent colon cleansing without tolerability and safety concerns continues to exist. Thirdly, most of colorectal surgery is conducted by using a laparoscopic technique. However, data on MBP in patients submitted for rectal surgery and laparoscopic colorectal surgery are not sufficient. Lastly, enhanced recovery after surgery (ERAS) is well established and has been developed to optimize perioperative care and to facilitate discharge. According to ERAS society's recommendations, preoperative MBP should not be used routinely in colorectal surgery [4]. In addition, the application of this recommendation to Korean patients must be verified.

In conclusion, more convincing data are required if $\mathrm{MBP}$ is to be abandoned. As the authors point out, members of the Korean Society of Coloproctology should make an effort to establish Korean guidelines for the use MBP. Large-scale prospective randomized trials should be conducted to achieve this purpose.

\section{REFERENCES}

1. Guenaga KF, Matos D, Wille-Jorgensen P. Mechanical bowel preparation for elective colorectal surgery. Cochrane Database Syst Rev 2011;(9):CD001544.

2. Zmora O, Wexner SD, Hajjar L, Park T, Efron JE, Nogueras JJ, et al. Trends in preparation for colorectal surgery: survey of the members of the American Society of Colon and Rectal Surgeons. Am Surg 2003;69:150-4.

3. Kang BM, Lee KY, Park SJ, Lee SH. Mechanical bowel preparation and prophylactic antibiotic administration in colorectal surgery: a survey of the current status in Korea. Ann Coloproctol 2013; 29:161-7.

4. Gustafsson UO, Scott MJ, Schwenk W, Demartines N, Roulin D, Francis N, et al. Guidelines for perioperative care in elective colonic surgery: Enhanced Recovery After Surgery (ERAS ${ }^{\circledast}$ ) Society recommendations. Clin Nutr 2012;31:783-800. 